\title{
Modeling anomalous heat diffusion: Comparing fractional derivative and non-linear diffusivity treatments.
}

\author{
F. Falcini ${ }^{1}$, R. Garra ${ }^{2}$, V. Voller ${ }^{3}$
}

October 8, 2018

\author{
${ }^{1}$ CNR-ISAC, Via Fosso del Cavaliere 100, 00133 Rome, Italy \\ ${ }^{2}$ Basque Center for Applied Mathematics, Mazarredo 14, 48009 Bilbao, \\ Spain \\ ${ }^{3}$ Department of Civil, Enviromental,and Geo- Engeneering, St. Anthony \\ Falls Laboratory, 500 Pillsbury Drive SE, Minneapolis, MN 55455, USA
}

\begin{abstract}
In the Fourier heat conduction equation, when the flux definition is expressed as the product of a constant diffusivity and the temperature gradient, the characteristic length scale evolves as the square root of time. However, if we replace the $1^{\text {st }}$ order transient and gradient terms in the Fourier equation with fractional derivatives and/or define a non-linear spatially dependent diffusivity, it is possible to generate an anomalous space-time scaling, i.e., a scaling where the time exponent differs from the expected value of $1 / 2$. To compare and contrast the possible consequences of using fractional calculus along with a non-linear flux, we investigate a space-time fractional heat diffusion equation that involves a non-linear diffusivity. Following presentation of the governing non-linear fractional equation, we arrive at a spacetime scaling that accounts for the combined anomalous contributions of memory (fractional derivative in time), non-locality (fractional derivative in space), and a non-linear diffusivity. We demonstrate how this scaling can manifest in a physical setting by considering the analytical solution of a non-linear fractional space-time diffusion equation, a limit case Stefan problem related to moisture infiltration into a porous media. A direct physically realizable simulation of this process shows how the anomalous space-time scaling is explicitly related to measures of both the memory and non-linearity in the system. Overall, the finding
\end{abstract}


from this work clearly shows how the definition of a non-linear diffusivity might contribute to anomalous diffusion behavior and suggests that, in modeling a particular observation, the roles of fractional derivatives and a suitably defined non-linear diffusivity are interchangeable.

\section{Introduction}

The classic one-dimensional heat transfer equation is the Fourier heat conduction equation

$$
\frac{\partial T}{\partial t}=\frac{\partial}{\partial x}\left(D \frac{\partial T}{\partial x}\right), x>0
$$

where $T$ is temperature and $D$ is the diffusivity. If we were to introduce the following space $x^{*}=x / \ell$ and time $t^{*}=t / \tau$ we can, on substitution into eq.(1), readily recover the well known square root relationship between the space and time scale, i.e., $\ell \sim \tau^{\frac{1}{2}}$. There has, however, been interest in studying so-called anomalous diffusion phenomena in heat transfer applications $[1,2,3,4,5,6,7]$. Such phenomena arise in situations where the problem domain includes heterogeneities with a power law distribution of length scales. Diffusion in the presence of these heterogeneities can exhibit "hold ups" or "fast paths" $[8,9,10]$, which disrupt the classic and expected square root of time dependence, i.e., $\ell \sim \tau^{n}, n \neq \frac{1}{2}$.

One way of modeling anomalous diffusion processes is through the use of fractional calculus $[10,11,12]$. Here the idea is to replace the transient and space gradient derivatives in the Fourier heat conduction with derivatives with fractional orders between 0 and 1; essentially a fractional in time derivative is formed by a convolution in time (representing memory effects) and a fraction in space derivative is formed as a convolution in space (representing non-locality). The analytical study of a limit case one-dimensional Stefan melting problem [4] has shown that when fractional time and space derivatives are employed in the governing equation the advance of the melt front goes as $s(t) \sim t^{\frac{\gamma}{1+\alpha}}$, where $0<\alpha \leq 1$ is the order of the space derivative and $0<\gamma \leq 1$ is the order of the time derivative; a scaling consistent with the known form for the fractional space-time diffusion equation [10]. Note with this scaling the choice of integer orders $(\alpha, \gamma=1)$ will give the standard relationship for the melt front, i.e., $s \sim t^{\frac{1}{2}}$, while with other appropriate choices of the derivative orders it is possible to generate exponents in the $s \sim t^{n}$ relationship, anywhere in the range $(0,1]$, where $n=\frac{\gamma}{1+\alpha}$. Also note that, memory effects - represented by fractional time derivatives - tend to induce so called sub-diffusion $n<\frac{1}{2}$ and non-local effects - represented by 
fractional space derivative - tend to induce so called super-diffusion $n>\frac{1}{2}$. Such anomalous behaviors of the front evolution, however, can also be generated by introducing non-linearity. For example, in studying a onedimensional Stefan melting problem, based on the integer derivative Fourier equation with the non-linear space dependent diffusivity $D \sim x^{\frac{2 \beta-1}{\beta}}, 0<$ $\beta \leq 1$, Falcini and Voller [13] obtain an analytical solution for the movement of the melt front that goes as $s \sim t^{\beta}$; i.e., an appropriate non-linear term in an integer derivative governing equation can generate the same anomalous diffusion signals (time exponent) as a governing heat diffusion equation with factional time and space derivatives.

Here our intentions are two fold. In the first place we will fully investigate the possible interchangeable contributions to anomalous diffusion signals through memory (realized through a fractional time derivative), nonlocality (realized through a fractional time derivative), and non-linear (realized through a non-linear diffusivity). In the second place we will provide a direct physical example that demonstrates how anomalous diffusion signals can be generated by a combination of memory and non-linear effects. In pursuing our first objective, after carefully defining the appropriate mathematical constructs, we will arrive at a heat diffusion governing equation that incorporates, in a general fashion, memory, non-local, and non-linear effects. A scaling analysis of this equation, supported by a closed form solution of a limit case Stefan problem, clearly reveals how the combination of memory, non-locality, and a non-linear diffusivity combine and compete to determine the time exponent in the anomalous diffusion signal. For our second objective we will appeal to recent experiments and simulations of horizontal infiltration $[14,15,16]$ - a problem directly related to our a limit case Stefan problem. In particular we will present a direct simulation of horizontal ( $x$ direction) infiltration into a porous medium containing a power-law (fractal) distribution of different sized flow obstacles, the fluidic conductivity between the obstacles increasing as a power-law in $x$.

\section{Definitions of non-locality, non-linearity, and mem- ory}

We start by writing the heat transfer equation in the half space $x>0$ as

$$
\frac{\partial u}{\partial t}=-\frac{\partial q}{\partial x}
$$


where $q(x, t)$ is the heat flux. In a basic treatment we expresses the flux $q$, at a given point, in terms of the temperature gradient at that point,

$$
q(x, t) \sim-\frac{\partial u}{\partial x} .
$$

This local treatment will recover the classical integer derivative (local) form of the Fourier heat conduction equation, eq.(1). By contrast, considering only up-stream contributions, a non-local flux definitions can be defined by the following convolution (e.g., $[11,12])$

$$
q^{\text {non-local }}(x, t) \sim-\int_{0}^{x}(x-\xi)^{-\alpha} \frac{\partial u}{\partial x}(\xi, t) d \xi, \quad \alpha \in(0,1]
$$

which, in a physical sense, states that the flux at a given point is a weighted sum of upstream gradients; $0<\alpha \leq 1$ is the degree of non-locality, which is related to the range of heterogeneity of the system. We notice that equation (4) is essentially a left-hand Caputo fractional space derivative of order $\alpha$, i.e., $q^{\text {non-local }} \sim-\partial^{\alpha} u / \partial x^{\alpha}$.

The local flux terms within the convolution of eq. (4) assume a linear diffusivity. This feature can be generalized by using a power-law in space diffusively $D \sim x^{\frac{2 \beta-1}{\beta}}, \quad 0<\beta \leq 1$ (e.g., a functionally graded material) to arrive at the following definition of a non-local, non-linear (NLNL) flux

$$
q^{N L N L} \sim-\int_{0}^{x}(x-\xi)^{-\alpha} x^{\frac{2 \beta-1}{\beta}} \frac{\partial u}{\partial \xi} d \xi
$$

a general treatment that recovers a purely non-local definition on setting $\beta=1 / 2$ and purely non-linear definition on setting $\alpha=1$. On defining the function $U(x, t)$ through

$$
\frac{\partial U}{\partial x}=-x^{\frac{2 \beta-1}{\beta}} \frac{\partial u}{\partial x}
$$

we can write down this general non-local, non-linear flux in the more compact form

$$
q^{N L N L} \sim-\frac{\partial^{\alpha} U}{\partial x^{\alpha}}
$$

Finally we note that, In addition to a non-local treatment through a fractional derivative in space, we can also introduce memory into our governing equation by replacing the time derivative in (1) with the Caputo fractional time derivative $[10,11,12]$

$$
\frac{\partial^{\gamma} u}{\partial t^{\gamma}} \sim \int_{0}^{t}(t-\tau)^{-\gamma} \frac{\partial u}{\partial \tau} d \tau, \quad \gamma \in(0,1]
$$


Finally, we note that we could also make further generalization of our non-local and memory models by allowing the orders of the fraction derivatives to be functions of the independent variables, so-called variable order fractional derivatives $[17,18,19]$. Here, however, we feel that our current purpose of comparing and contrasting fractional derivative and non-linear treatments of diffusion transport is better made by assuming that our fractional space and time orders $(\beta$ and $\gamma)$ are constant.

\section{$3 \quad$ Space-time scaling}

Utilizing the modeling options of memory, non-locality, and a non-linear flux, allows us to construct a general heat conduction equation with the form

$$
\frac{\partial^{\gamma} u}{\partial t^{\gamma}}=\frac{\partial}{\partial x}\left(\frac{\partial^{\alpha} U}{\partial x^{\alpha}}\right)
$$

We introduce the following scalings $t^{*}=t / \tau, x^{*}=x / \ell, u^{*}=u / u_{\text {ref }}, U^{*}=$ $U / U_{\text {ref }}$, where $\tau, \ell$ are time and space scales, respectively, and $u_{r e f}, U_{r e f}$ are reference values; note, without loss of generality, we will set $u_{r e f}=1$. When these scalings are used in eq.(9) we obtain the following relation between the space and time scales

$$
\begin{gathered}
\tau^{-\gamma} \sim U_{r e f} \ell^{-1-\alpha}, \\
U_{r e f} \sim \ell^{\frac{2 \beta-1}{\beta}},
\end{gathered}
$$

which on combination lead to the following space-time scaling

$$
\ell \sim \tau^{n}, n=\frac{\gamma}{[1+\alpha]+\left[\left(\frac{1}{\beta}-2\right)\right]},(\alpha, \beta, \gamma) \in(0,1]
$$

for the space-time fractional diffusion equation with a non-linear flux.

To categorize the different anomalous behaviors that can result from the values of $\alpha, \gamma, \beta$ we consider three possible outcomes from our scaling analysis of eq.(12), sub- $(n<1 / 2)$, super- $(1 / 2<n<1)$, and super ${ }^{+}$. $(n>1)$. The results using this nomenclature are summarized in Table 1. In the first row of the table we show that the settings $\alpha=1, \beta=1 / 2, \gamma=$ 1 recovers the base case of a memory free, local, linear equation with no anomalous transport behaviors. In rows 2-4 we consider changes in the range $(0,1]$ for each parameter in turn, while fixing the remaining parameters at their "normal diffusion values" ( $\alpha=1, \beta=1 / 2, \gamma=1)$. The striking 
feature is that, while the fractional derivative treatments are restricted to either sub- $n<1 / 2$ (memory) and super- $n>1 / 2$ (non-local) behaviors, the appropriate choice of the non-linear parameter $\beta$ spans the whole subballistic range $(0<n<1)$. This suggests that a non-linear treatment could be used in place of a non-local or memory fractional derivative treatment.

To gain yet further insight we also consider varying pairs of parameters ([non-local, memory], [non-linear, memory], and [non-local, non-linear]) while keeping the remaining parameter fixed at the "normal" value. The last three rows of Table 1 shows the types of anomalous diffusion behaviors that can result from these pairings. We note that the case of [non-local, memory] behaviors (row 5) recovers the scaling $n=\frac{\gamma}{1+\alpha}$ previously derived by Metzler and Klafter [10] for the fractional transient diffusion equation with a linear diffusion coefficient. When we compare this scaling to the [non-linear only] behaviors (row 4) we see that a non-linear diffusivity treatment could be used in place of any combination of non-local and memory fraction derivative treatments. Further, we also note that the combination of non-local and non-linear treatments (row 7 ) allows for the possibility of time exponents in excess of the ballistic limit, i.e., value of $n>1$.

\section{Manifestation of space-time scaling in a heat/mass transfer setting}

The analysis above is very much in the abstract. To move away from this we need to answer the question in what physically realizable systems should one expect to see anomalous diffusion behaviors? To answer this question we consider a limit case Stefan melting problem related to the infiltration of moisture into an initially empty porous tube. In previous work Voller [4] showed that this problem arrises in the case when the specific heat in a one-phase Stefan melting problem is allowed to go to zero. In that work Voller developed a fractional derivative version of the governing equations and examined how the exponent $n$ in the closed form solution for determining the movement of the moisture front $s=t^{n}$ could - depending on non-locality $0<\alpha \leq 1$ and memory $0<\gamma \leq 1$ - take on sub-diffusive $(n<1 / 2)$ and super-diffusive $(n>1 / 2)$ values. Here we look at the alternative problem where we have a local flux definition $(\alpha=1)$ but account for memory $(0<$ $\gamma \leq 1$ ) and a non-linear power-law diffusivity $D \sim x^{\frac{2 \beta-1}{\beta}}, \quad 0<\beta \leq 1$. In this way, our governing equation is a steady state problem in the growing 
Table 1: Range of Anomalous Behaviors Through Choice of Values for the Non-locality $0<\alpha \leq 1$, Memory $0<\gamma \leq 1$, and Non-linearity $0<\beta \leq 1$

\begin{tabular}{|l|c|c|c|c|}
\hline type & exponent & sub- & super- & super $^{+}$ \\
\hline \hline $\begin{array}{l}\text { normal } \\
\alpha=1, \beta=0.5, \gamma=1\end{array}$ & $n=\frac{1}{2}$ & No & No & No \\
\hline $\begin{array}{l}\text { non-local only } \\
\alpha \in(0,1]\end{array}$ & $n=\frac{1}{1+\alpha}$ & No & Yes & No \\
\hline $\begin{array}{l}\text { memory only } \\
\gamma \in(0,1]\end{array}$ & $n=\frac{\gamma}{2}$ & Yes & No & No \\
\hline $\begin{array}{l}\text { non-linear only } \\
\beta \in(0,1]\end{array}$ & $n=\beta$ & $\beta \in(0,0.5]$ & $\beta \in(0.5,1]$ & No \\
\hline $\begin{array}{l}\text { non-local }+ \text { memory } \\
\alpha, \gamma \in(0,1]\end{array}$ & $n=\frac{\gamma}{1+\alpha}$ & $1+\alpha>2 \gamma$ & $1+\alpha<2 \gamma$ & No \\
\hline $\begin{array}{l}\text { non-linear }+ \text { memory } \\
\beta, \gamma \in(0,1]\end{array}$ & $n=\beta \gamma$ & $\beta \gamma<1 / 2$ & $\beta \gamma>1 / 2$ & No \\
\hline $\begin{array}{l}\text { non-local }+ \text { non- } \\
\text { linear } \\
\alpha, \beta \in(0,1]\end{array}$ & $n=\frac{1}{\alpha+\frac{1}{\beta}-1}$ & $\alpha+\frac{1}{\beta}>3$ & $3>\alpha+\frac{1}{\beta}>2$ & $\alpha+\frac{1}{\beta}<2$ \\
\hline
\end{tabular}

domain $(0, s(t) \geq 0)$

$$
\frac{\partial}{\partial x}\left(x^{\frac{2 \beta-1}{\beta}} \frac{\partial u}{\partial x}\right)=0, \quad x \in(0, s(t))
$$

with boundary conditions $u(0)=1$, and $u(s)=0$. The time evolution of the system is introduced by assuming that the moving boundary $s(t)$ satisfies the Stefan-like melting condition

$$
-\left(x^{\frac{2 \beta-1}{\beta}} \frac{\partial u}{\partial x}\right)_{x=s}=\frac{d^{\gamma} s}{d t^{\gamma}}
$$

The solution of eq.(13) that satisfies the boundary conditions is

$$
u=1-\left(\frac{x}{s}\right)^{\frac{1-\beta}{\beta}} \text {. }
$$

When this solution is used in our Stefan condition eq.(14) we get

$$
s^{\frac{1-\beta}{\beta}} \sim \frac{d^{\gamma} s}{d t^{\gamma}}, \quad s(0)=0 .
$$




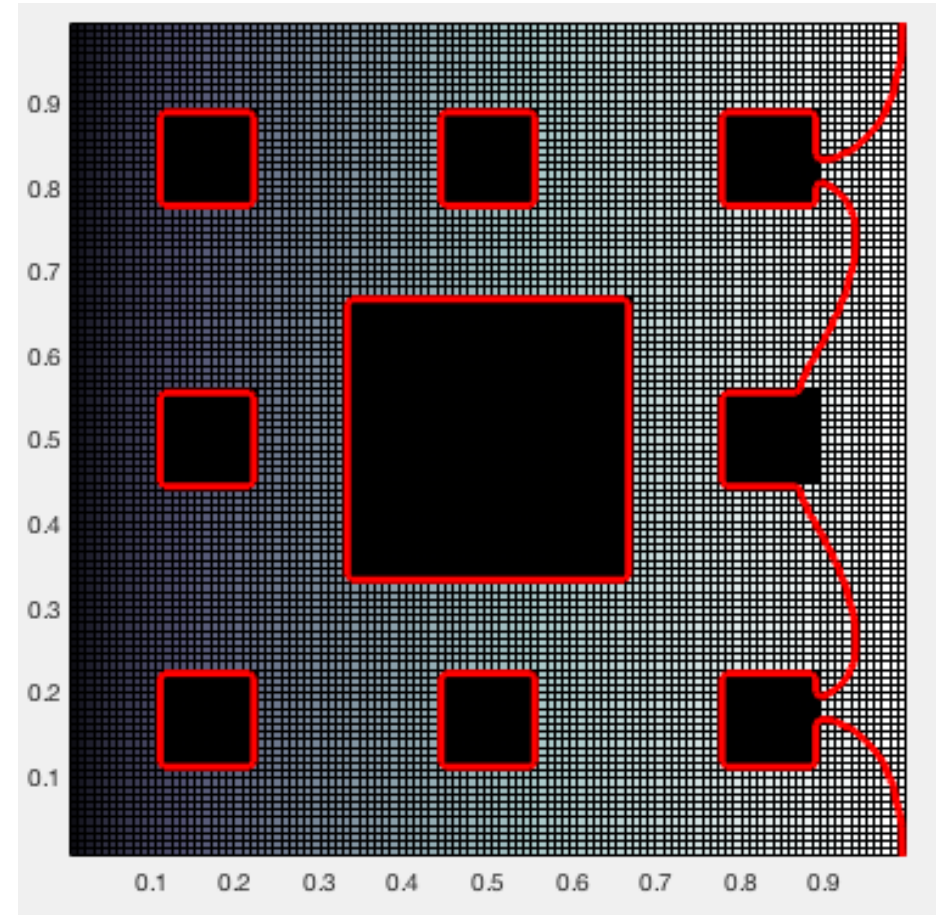

Figure 1: Infiltration into a porous medium containing impermeable inclusions (black squares) laid out as a Serpinski fractal carpet. The background diffusivity is increasing as a power-law with increasing position $x$, (darker to lighter). The image shown, is at the point where the infiltration fluid has reached the end of the domain - the red line indicates the position of the filling front.

Noting that when $n>0$ the Caputo fractional derivative $\frac{\partial^{\gamma}}{\partial t^{\gamma}} t^{n} \sim t^{n-\gamma}[11]$, suggests the following analytical form for the advance of the moisture front

$$
s(t)=t^{n}, n=\beta \gamma
$$

exactly the exponent we would expect from our scaling analysis in eq.(12) (see row 6 in Table 1 ).

While the above closed form solution demonstrates how our proposed scaling emerges from a non-linear fractional treatment applied to an infiltration problem it does not provide a physical justification for using such a model. To mitigate this shortcoming and to complement our analysis, we 
will present a direct simulation that explicitly demonstrates how our scaling emerges from a physically realizable infiltration scenario.

In physical applications, our general, one-dimensional, non-linear fractional derivative equation (eq. (9)) can be used, after appropriate averaging, as a model for predominately uni-directional transport through a multi-dimensional heterogeneous domain. With this in mind, for our physical demonstration, we will consider infiltration into a unit square domain $(x \in[0,1], y \in[0,1])$, containing an initially empty and dry (air filled) heterogenous porous medium; the heterogeneity, induced by impermeable inclusions, laid out as a truncated fractal (Serpinski) carpet, see Fig. 1. In the simulation we assume that fluid is driven into the domain by the application of a fixed head, $u=1$, along the $y$-oriented boundary at $x=0$. Moreover, we (i) impose no flow across the $x$-oriented boundaries; (ii) assume that there is a sharp interface between the infiltrating fluid and unfilled parts of the domain; (iii) set, at each point in time, a gauge pressure head $u=0$, in the dry regions.

According to the proposed setting (Fig. 1), the presence of impermeable inclusions would require a two-dimensional treatment. However, we will be primarily interested in calculating an infiltration length $s(t)$, defined as the equivalent $x$-length of fluid in the domain. This value is readily evaluated by calculating, at any point in time, the area fraction of the domain covered by fluid. Our contention is that due to its one-dimensional nature, the time evolution of the infiltration length can be modeled by the fractional governing infiltration equations, given by (13) and (14).

The simulation is performed by means of a volume of fluid (vof) method [20] - full details of the approach, along with empirical validation are given in $[14,15,16]$. In this previous work the simulations considered cases where the background diffusivity — the fluidic conductivity of the medium in the spaces between the fractal carpet flow obstacles - is a fixed constant, e.g., $k=1$. Results showed that the evolution of the infiltration length was an anomalous sub-diffusion with a time exponent less than the square root, i.e., $s \sim t^{n}, t<1 / 2$. Concurrent scaling work [21] determined that the expected value of the time exponent is related to properties of the fractal carpet of the inclusions, i.e., $n=(H-1) \nu$, where, for the carpet pattern in Fig. $1, H=1.89<2$ is the fractal dimension and $\nu=0.475<0.5$ is the time exponent associated with a spreading random walker in the spaces between the obstacles (impermeable inclusions). We note that this sub-diffusive nature of the evolution of the infiltration length $s(t)$ can be realized by setting the time derivative order $\gamma=2 n=0.846$ in our proposed fractional derivative infiltration model, viz., eqs.(13) and (14). 
Here we extend the previous works by Voller and co-workers $([14,15,16])$, by assuming that the background fluidic conductivity is not unity but has the power-law dependence $k=x^{\frac{1}{2}}$. With reference to the non-linear diffusive form in eq.(6), this choice implies that $\beta=\frac{2}{3}$. If our scaling is applicable, the direct vof simulation of infiltration into this porous medium should generate a supper-diffusive time behavior for the infiltration length, i.e., $s(t)=t^{n}, n=$

$\gamma \beta=\frac{2}{3} \times 0.846=0.564$. Figure 2 shows a plot of the simulated predictions of $\log s$ vs. $\log t$. This plot is close to linear, indicating a power law dependence of infiltration length $s$ on time $t$. Moreover, the best fit line through the data has a slope of $n=0.564$, exactly matching our scaling prediction from the solution of eqs. (13) and (14). Thus, we have demonstrated how our suggested scaling for a problem with memory and non-linearity can be manifest in a physically realizable system. Interestingly, in this case, the contributions of the non-linearity and memory to the final time exponent can be determined a-priori from the form of the non-linearity and the geometry of the obstacle field.

\section{Conclusion}

The core result in this work is the scaling of non-linear, space-time fractional heat diffusion, i.e., eq.(12), extending the known scaling associated with linear space-time fractional diffusion [10]. We have demonstrated, both by considering the analytical solution of a limit case Stefan problem and through direct simulation, how this scaling can manifest in realistic heat/mass transfer applications. This analysis is worthwhile because it provides us a possible means of interpreting the physical cause of observed anomalous diffusion signals. We need, however, to take care with such an analysis. While our direct simulation clearly demonstrates how the individual parameters of a non-linear diffusivity, memory (fractional time derivative), and non-locality (fractional space derivative) in the transport model can combine together to arrive at the time exponent in the space time scaling, we should be aware that an appropriate non-linear flux definition will always be able to recover the full range $0<n \leq 1$ of anomalous diffusion behaviors. So in every case, we need to debate, if an anomalous diffusion signal genuinely includes memory and non-local effects or if it is the result of a non-linear flux alone. 


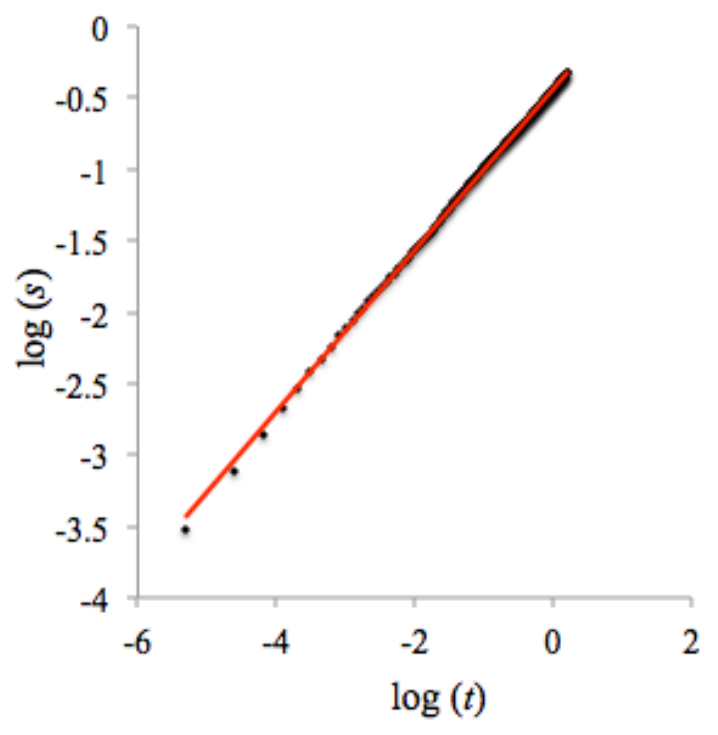

Figure 2: log-log plot of predicted infiltration length $s$ against time $t$ for the porous medium in Fig. 1, shown as black dots. Due to the combination of memory $(\gamma=0.846)$ and non-linear diffusivity $\left(\beta=\frac{2}{3}\right)$ the best fit power law (red line) though the predicted data has a slope (time exponent) of $n=\gamma \beta=0.564$.

\section{Acknowledgements}

This research is supported by the Basque Government through the BERC 2014-2017 program and by Spanish Ministry of Economy and Competitiveness MINECO: BCAM Severo Ochoa excellence accreditation SEV-20130323 .

\section{References}

[1] Li, X., Xu, M., and Wang, S. (2008). Scale-invariant solutions to partial differential equations of fractional order with a moving boundary condition, Phys. A: Math. Theor., 41, 155202, pp. 11. 
[2] Liu, J., and Xu, M. (2009). Some exact solutions to Stefan problems with fractional differential equations, J. Math. Anal. Appl., 351, 536542 .

[3] Atkinson, C. (2012). Moving boundary problems for time fractional and composition dependent diffusion, Fractional Calculus Appl. Anal., 15, 207-221.

[4] Voller, V. R. (2010). An exact solution of a limit case Stefan problem governed by a fractional diffusion equation,International Journal of Heat and Mass Transfer, 53(23), 5622-5625.

[5] Voller, V. R., Falcini, F., and Garra, R. (2013). Fractional Stefan problems exhibiting lumped and distributed latent-heat memory effects, Physical Review E, 87(4), 042401.

[6] Voller, V.R. (2014). Fractional Stefan Problems, International Journal Heat Mass Transfer, 74, 269-277.

[7] Voller, V.R. (2016). Computations of anomalous phase change, International Journal of Numerical Methods Heat Fluid Flow, $26(3 / 4)$, 624-638.

[8] Zhang, Y., Benson, D. A., and Reeves, D. M. (2009). Time and space non-localities underlying fractional-derivative models: Distinction and literature review of field applications, Advances in Water Resources, $32(4), 561-581$.

[9] Schumer, R., Meerschaert, M. M., and Baeumer, B. (2009). Fractional advection-dispersion equations for modeling transport at the Earth surface, Journal of Geophysical Research: Earth Surface, 114(F4)

[10] Metzler, R., and Klafter, J. (2000). The random walk's guide to anomalous diffusion: a fractional dynamics approach, Physics reports, 339(1), $1-77$.

[11] Podlubny, I. (1998). Fractional differential equations: an introduction to fractional derivatives, fractional differential equations, to methods of their solution and some of their applications (Vol. 198). Academic press.

[12] Meerschaert, M. M., and Sikorskii, A. (2012). Stochastic models for fractional calculus, (Vol. 43). Walter de Gruyter. 
[13] Voller, V. R., and Falcini, F. (2013). Two exact solutions of a Stefan problem with varying diffusivity, International Journal of Heat and Mass Transfer, 58(1), 80-85.

[14] Voller, V.R. (2015). A direct simulation demonstrating the role of spacial heterogeneity in determining anomalous transport, Water Resources Research, 51, 2119-2127.

[15] Filipovitch, N., Hill, K.M., Longjas, A., and Voller, V.R. (2016), Infiltration experiments demonstrate an explicit connection between heterogeneity and anomalous diffusion behavior, Water Resources Research, $52,5167-5178$.

[16] Aarão Reis, F.D.A., Bolster, D., and Voller V.R. (2018), Anomalous behaviors during infiltration into heterogeneous porous media, Advances Water Resources, 113, 180-188.

[17] Valério, D. and Sá da Costa. J. (2011). Variable-order fractional derivatives and their numerical approximations, Signal Processing, 91, 470483.

[18] Fu, Z-J., Chen, W., and Ling, L. (2015). Method of approximate particular solutions for constant- and variable-order fractional diffusion models, Engineering Analysis with Boundary Elements, 57, 37-46.

[19] Tavares, D., Almeida, R., and Torres, D.F.M. (2016). Caputo derivatives of fractional order: Numerical approximation, Commun. Nonlinear Sci, Numer. Simulat., 35, 69-87.

[20] Hirt, C.W. and Nichols, B.D. (1981). Volume-of Fluid (VOF) method for the dynamics of free boundaries, J. Comput. Phys, 39, 201-225.

[21] Aarão Reis, F.D.A. (2016). Scaling relations in the diffusive infiltration in fractals, Physical Review E, doi:10.1103/PhysRevE.94.052124. 